\title{
RAPID UNCERTAINTY QUANTIFICATION OF THE STABILITY ANALYSIS USING A PROBABILISTIC ESTIMATION OF THE PROCESS FORCE PARAMETERS
}

\author{
M. Busch ${ }^{1 *}$, B. Schmucker ${ }^{1}$, M. F. Zaeh ${ }^{1}$ \\ ${ }^{1}$ Technical University of Munich, Institute for Machine Tools and Industrial Management, Garching near Munich, \\ Germany \\ *Corresponding author; e-mail: maximilian.busch@iwb.tum.de
}

\begin{abstract}
In the course of the digitization of modern production systems, a reliable parameterization of the digital twin of machining processes is essential. For example, the digital representation of milling operations enables the process parameter selection without time-consuming and expensive test series by using stability lobe diagrams (SLD). However, the parameterization of the underlying process force model with very few cutting force experiments can prevent a reliable process design, as errors in the parameterization process are propagated to the stability analysis.

Therefore, a novel two-step methodology is proposed to provide probabilistic credible intervals for conventional stability lobe diagrams: First, the unknown parameters of the process force model are estimated using a Bayesian regression method. Secondly, the estimated probability distributions of the process force parameters are used to quantify the uncertainty of the stability boundary using a mechanistic process force model.

The proposed methodology is particularly characterized by its low computational cost, since timeconsuming and computationally expensive Monte Carlo procedures are avoided. Instead, the methodology relies on the analytical derivation of the model parameters' posterior probability distribution and on polynomial chaos expansion (PCE) algorithms to quantify the uncertainty in the final stability analysis.
\end{abstract}

\section{Keywords:}

Uncertainty quantification; probabilistic parameter estimation; machining simulation; stability analysis

\section{INTRODUCTION}

The efficiency of modern production processes, such as milling, is heavily dependent on a robust process stability. These production processes can be changed, adapted and reconfigured due to modern cyber-physical systems [Schmucker et al. 2021]. Therefore, the simulation-based, predictive analysis of the process stability without timeconsuming test series is increasingly important and essential.

The underlying force models are mostly based on a few parameters, whose identification by experimental force measurements is often subject to errors. Hence, it is crucial to quantify the parameter uncertainties which may arise due to an uncertain parameter estimation procedure. Subsequently, the following model-based stability estimation is also prone to uncertainty.

Recent works on probabilistic programming to estimate cutting force model parameters and on uncertainty quantification of stability limits show that such an uncertainty quantification methodology is reasonable, but due to its algorithmic structure computationally expensive and cumbersome.

In order to provide a stability analysis, which is reliable and fast at the same time and thus can be used on a regular basis for modern reconfigurable production systems, a novel methodology to quantify the arising uncertainty in a stability analysis is proposed. The methodology relies on the analytic computation of the probabilistic regression to identify uncertain cutting force coefficients, whereas the following uncertainty quantification of the stability limit is based on polynomial chaos expansions to reduce the computational burden of the uncertainty propagation scheme. Figure 1 illustrates the proposed methodology. 


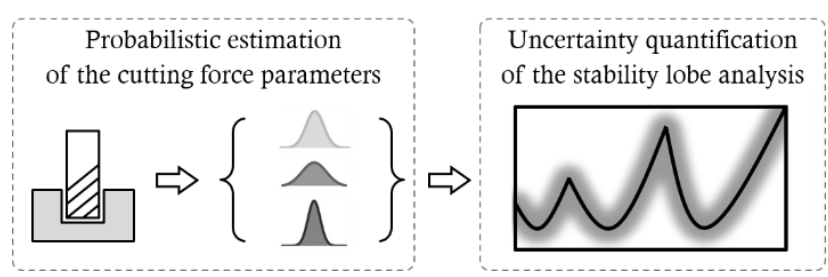

Figure 1: Methodology to quantify the uncertainty of stability analyses based on a probabilistic parameter estimation of the cutting force coefficients.

This paper is structured as follows:

First, recent works on probabilistic cutting force parameter estimation and a subsequent uncertainty quantification of the stability limit are presented in section 2 . Thereafter, a novel probabilistic approach to estimate the cutting force parameters is presented in section 3 . In section 4 , the uncertainty in the cutting force parameters is propagated to the stability analysis using non-sampling based algorithms. The implementation details are presented in section 5 , whereas the results are discussed in section 6 . Section 7 summarizes and concludes this paper.

\section{FUNDAMENTALS AND RECENT WORK}

Usually, the stability analysis of conventional milling processes is based on the approach of Altintas and Budak [Altintas 2001; Altintas et al. 1995], resulting in the wellknown stability lobe diagrams. The stability limit, which is characterized by the chatter free axial depth of cut $b_{\text {lim }}$, can be calculated as follows:

$$
b_{\text {lim }}=-\frac{2 \pi \Lambda_{R}}{N_{t} K_{t}}\left(1+\kappa^{2}\right) .
$$

$N_{t}$ is the number of teeth and $K_{t}$ is a cutting factor. $\kappa$ is defined as

$$
\kappa=\frac{\Lambda_{I}}{\Lambda_{R}}
$$

$\Lambda_{R}$ and $\Lambda_{I}$ are the real and imaginary parts of the eigenvalue $\Lambda$, which is calculated by solving the characteristic eigenvalue problem

$$
\operatorname{det}\left(I+\Lambda G_{0}\left(i \omega_{c}\right)\right)=0 .
$$

$G_{0}\left(i \omega_{c}\right)$ is defined as the oriented transfer function matrix as described in detail in [Altintas et al. 1995]. Besides a given chatter frequency $\omega_{c}$ and the radial immersion angles $\phi_{s}$ and $\phi_{e}$, the oriented transfer function matrix is dependent on the cutting factors $K_{t}$ and $K_{r}$.

Finally, these cutting factors can be determined using experimental cutting force measurements and linear regression techniques.

Exemplarily for full immersion cuts (slotting), the average cutting forces in feed and normal direction are modelled as follows [Schmitz et al. 2019]:

$$
\bar{F}_{\text {Feed }}=\frac{N_{\mathrm{t}} b k_{\mathrm{n}}}{4} f_{\mathrm{t}}+\frac{N_{\mathrm{t}} b k_{\mathrm{ne}}}{\pi},
$$

and

$$
\bar{F}_{\text {Normal }}=\frac{N_{\mathrm{t}} b k_{\mathrm{t}}}{4} f_{\mathrm{t}}+\frac{N_{\mathrm{t}} b k_{\mathrm{te}}}{\pi},
$$

where $b$ is the axial depth of cut and $f_{t}$ is the feed per tooth. Using multiple cutting force experiments with various feeds per tooth, the four cutting force coefficients $k_{t}, k_{n}, k_{t e}$ and $k_{n e}$ can be determined based on linear regression between the feed per tooth $f_{t}$ and the average cutting forces $\bar{F}_{F e e d}$ and $\bar{F}_{\text {Normal }}$. Figure 2 illustrates this approach.

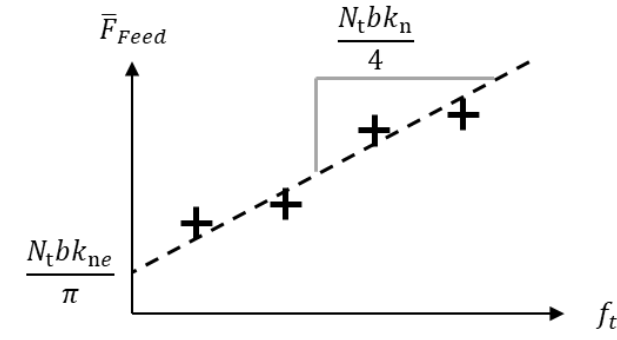

Figure 2: Principle scheme of the identification of $\boldsymbol{k}_{n}$ and $k_{n e}$ using a linear regression of experimental data (based on [Schmitz et al. 2019]).

Typically, the linear regression is estimated using ordinary least squares (OLS) methods, which minimize the total sum of squared residuals between the fitted line and the measured data points [Schmitz et al. 2019].

By using the OLS methods, the overdetermined linear regression models

and

$$
\overline{\boldsymbol{F}}_{\text {Feed }}=\boldsymbol{X}_{\text {Feed }} \cdot \boldsymbol{\beta}_{\text {Feed }}+\epsilon_{\text {Feed }}
$$

$$
\overline{\boldsymbol{F}}_{\text {Normal }}=\boldsymbol{X}_{\text {Normal }} \cdot \boldsymbol{\beta}_{\text {Normal }}+\epsilon_{\text {Normal }}
$$

can be solved. $\boldsymbol{X}_{\text {Feed }}$ and $\boldsymbol{X}_{\text {Normal }}$ represent the observations (also called design matrix), whereas $\boldsymbol{\beta}_{\text {Feed }}$ and $\boldsymbol{\beta}_{\text {Normal }}$ represent the four cutting coefficients:

$$
\begin{gathered}
\boldsymbol{\beta}_{\text {Feed }}=\left[\begin{array}{ll}
k_{n} & k_{n e}
\end{array}\right]^{T}, \\
\boldsymbol{\beta}_{\text {Normal }}=\left[\begin{array}{ll}
k_{t} & k_{t e}
\end{array}\right]^{T} .
\end{gathered}
$$

The cutting factors $K_{t}$ and $K_{r}$ used in the stability analysis can be directly computed from the estimated cutting force coefficients [Schmitz et al. 2019]:

$$
\begin{aligned}
& K_{t}=k_{t}, \\
& K_{r}=\frac{1}{\tan \alpha},
\end{aligned}
$$

with

$$
\alpha=\tan ^{-1}\left(\frac{k_{n}}{k_{t}}\right)
$$

If there are only few cutting experiments available, the estimated OLS solution may be prone to errors.

In order to compensate for those errors and to deal with the resulting uncertainty, multiple probabilistic approaches have been proposed to estimate machining force parameters using Bayesian inference techniques [Bhattacharyya et al. 2021; Karandikar et al. 2014; Salehi et al. 2019]. Bayesian inference methods are increasingly popular to estimate probability distributions of model parameters. Therefore, the model parameters are not considered to be deterministic and constant point estimates, but rather represented by random variables with an underlying probability distribution. Using Bayes Theorem, this prior belief on the parameter distributions can be updated by considering a limited number of experimental observations.

As the inference of the underlying probability distributions is often computationally not solvable, numerical Markov Chain Monte Carlo (MCMC) sampling techniques are used to infer the underlying distributions of the cutting force parameters [Karandikar et al. 2014]. However, as MCMC techniques make use of Monte Carlo sampling techniques, the inference of the probability distributions remains computationally intensive.

As proclaimed by Schmitz and Smith [Schmitz et al. 2019], uncertainties in the cutting force coefficients result in uncertainties of the stability limits. Hence, similar to the 
Bayesian regression estimates, the stability limit $b_{\text {lim }}$ is not a deterministic result of a calculation, but also a random variable with an underlying probability distribution. Figure 3 illustrates this assumption.

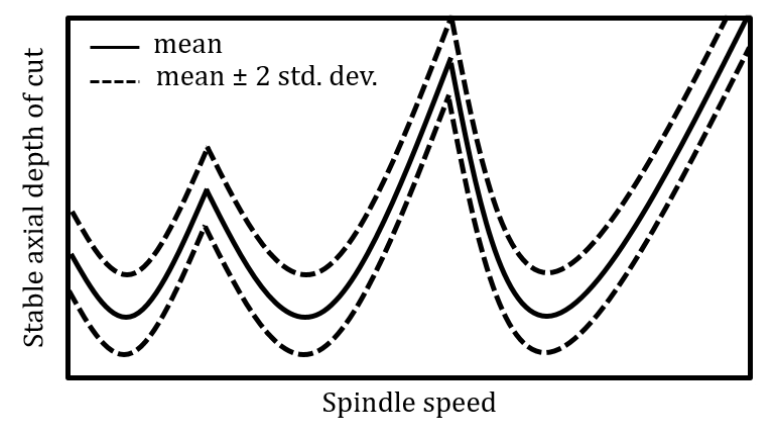

Figure 3: Principle scheme of uncertainty-based stability lobe diagrams (based on [Schmitz and Smith 2019]).

$$
p\left(\boldsymbol{\beta}, \sigma^{2}\right)=\frac{b^{a}}{(2 \pi)^{\frac{n}{2}}\left|\boldsymbol{V}_{\boldsymbol{\beta}}\right|^{\frac{1}{2}} \Gamma(a)}\left(\frac{1}{\sigma^{2}}\right)^{a+\frac{n}{2}+1} \cdot \exp \left[-\frac{1}{\sigma^{2}} \cdot\left\{b+\frac{1}{2}\left(\boldsymbol{\beta}-\boldsymbol{\mu}_{\boldsymbol{\beta}}\right)^{T} \boldsymbol{V}_{\boldsymbol{\beta}}^{-1}\left(\boldsymbol{\beta}-\boldsymbol{\mu}_{\beta}\right)\right\}\right]=N I G\left(\boldsymbol{\mu}_{\boldsymbol{\beta}}, \boldsymbol{V}_{\boldsymbol{\beta}}, a, b\right) .
$$

estimate the underlying probability distributions of the cutting force parameters. In comparison to Karandikar et al., who rely on computationally intensive MCMC algorithms, the Bayesian inference is chosen to rely on conjugate priors. In contrast to conventional MCMC approaches for Bayesian linear regression, conjugate priors provide an analytical solution for the posterior distributions. Therefore, conjugate normal priors with an inverse gamma error function are chosen. The choice of priors is well founded by the experiments and results presented in [Duncan et al. 2005; Karandikar et al. 2014].

Hence, the Bayesian linear regression problem is stated as follows, where $\boldsymbol{\beta}_{\text {Feed }}$ and $\boldsymbol{\beta}_{\text {Normal }}$ now represent the joint probability distributions of $\left[\begin{array}{ll}k_{n} & k_{n e}\end{array}\right]^{T}$ and $\left[\begin{array}{ll}k_{t} & k_{t e}\end{array}\right]^{T}$ :

$$
\begin{gathered}
\overline{\boldsymbol{F}}_{\text {Feed }} \sim \boldsymbol{N}\left(\boldsymbol{X}_{\text {Feed }} \cdot \boldsymbol{\beta}_{\text {Feed }}, \sigma_{\text {Feed }}^{2} \cdot \boldsymbol{I}\right), \\
\overline{\boldsymbol{F}}_{\text {Normal }} \sim \boldsymbol{N}\left(\boldsymbol{X}_{\text {Normal }} \cdot \boldsymbol{\beta}_{\text {Normal }}, \sigma_{\text {Normal }}^{2} \cdot \boldsymbol{I}\right) .
\end{gathered}
$$

Conjugate prior beliefs in form of Normal-Inverse-Gamma (NIG) priors are placed on the unknown cutting force coefficients in $\boldsymbol{\beta}_{\text {Feed }}$ and $\boldsymbol{\beta}_{\text {Normal }}$ (the directional indices Feed and Normal are left out for better readability):
Different approaches to estimate the underlying probability distributions of the stability limits have been proposed:

Duncan et al. [Duncan et al. 2005] proposed a methodology to infer the stability limits' uncertainty based on uncertain cutting force parameters and uncertain frequency response functions at the tool tip. Normal distributions were assumed for the cutting force parameters as well as for the frequency response functions. A conventional Monte Carlo simulation approach with 1000 samples was used to estimate the resulting uncertainty of the stability boundary.

Similarly, Li et al. [Li et al. 2020] proposed an MCMC-based inference framework to estimate the stability lobe uncertainty based on uniformly distributed cutting force coefficients and normally distributed modal parameters.

In contrast, Karandikar et al. [Karandikar et al. 2020] performed a Bayesian inference scheme directly for the stability lobe diagram. Thus, the identification of cutting force parameters is not required, but multiple stability measurements using different process parameters are needed to update the prior belief. The prior was modelled using a Monte Carlo simulation and normal distributions were assumed for the cutting force parameters, tool properties and stiffness and damping terms.

It is apparent, that the current methods to estimate the cutting force parameters as well as the current methods to propagate the uncertainty into the stability analysis heavily rely on Monte Carlo approaches. Therefore, their application in reconfigurable systems and production processes is limited as conventional Monte Carlo schemes are computationally expensive and cumbersome.

In order to solve this issue, a novel methodology for rapid uncertainty quantification of the stability analysis is proposed and described in the following sections 3 and 4 .

\section{BAYESIAN PARAMETER ESTIMATION}

Similarly to the approach presented by Karandikar et al. [Karandikar et al. 2014], a probabilistic inference scheme to estimate uncertain cutting force parameters is chosen. Furthermore, a Bayesian regression technique is used to
Such a prior is dependent on the mean $\boldsymbol{\mu}_{\boldsymbol{\beta}}$, the variance $\boldsymbol{V}_{\boldsymbol{\beta}}$, the shape parameters $a$ and $b$, the Gamma function $\Gamma(a)$ and the rank $n$ of the design matrix $\boldsymbol{X}$.

It can be shown, that by using the given prior with the given Gaussian likelihood function, the resulting posterior distributions $p\left(\boldsymbol{\beta}, \sigma^{2} \mid \overline{\boldsymbol{F}}\right)$ of the cutting force parameters are also in NIG form (the posterior quantities are denoted with $*)$ :

$$
p\left(\boldsymbol{\beta}, \sigma^{2} \mid \overline{\boldsymbol{F}}\right)=N I G\left(\boldsymbol{\mu}_{\boldsymbol{\beta}}^{*}, \boldsymbol{V}_{\boldsymbol{\beta}}^{*}, a^{*}, b^{*}\right) .
$$

Specifically, their posterior quantities can be analytically computed based on the prior assumptions by

$$
\begin{aligned}
\boldsymbol{\mu}_{\boldsymbol{\beta}}^{*} & =\left(\boldsymbol{V}_{\boldsymbol{\beta}}^{-1}+\boldsymbol{X}^{T} \boldsymbol{X}\right)^{-1}\left(\boldsymbol{V}_{\boldsymbol{\beta}}^{-1} \boldsymbol{\mu}_{\boldsymbol{\beta}}+\boldsymbol{X}^{T} \overline{\boldsymbol{F}}\right), \\
\boldsymbol{V}_{\boldsymbol{\beta}}^{*} & =\left(\boldsymbol{V}_{\boldsymbol{\beta}}^{-1}+\boldsymbol{X}^{T} \boldsymbol{X}\right)^{-1} \\
a^{*} & =a+\frac{N}{2} \\
b^{*} & =b+\frac{1}{2}\left[\boldsymbol{\mu}_{\boldsymbol{\beta}}^{T} \boldsymbol{V}_{\boldsymbol{\beta}}^{-1} \boldsymbol{\mu}_{\boldsymbol{\beta}}+\overline{\boldsymbol{F}}^{T} \overline{\boldsymbol{F}}-\boldsymbol{\mu}^{* T} \boldsymbol{V}^{*-1} \boldsymbol{\mu}^{*}\right]
\end{aligned}
$$

$N$ denotes the number of observations.

Due to the analytic solution, the Bayesian linear regression approach can be solved significantly faster than conventional MCMC approaches.

The approach was validated using three cutting force experiments conducted on a GROB G350 machine with a $16 \mathrm{~mm}$ end mill tool with a helix angle of $30^{\circ}$, three flutes and full immersion cuts in $\mathrm{C} 45 \mathrm{U}$ steel. The cutting forces in feed and normal direction were measured using a Kistler dynamometer.

Figure 4 illustrates the resulting Bayesian linear regression based on the conjugate prior assumption. The estimated means $\mu_{\text {Feed }}$ and $\mu_{\text {Normal }}$ and their $95 \%$ credible intervals are depicted as well. 

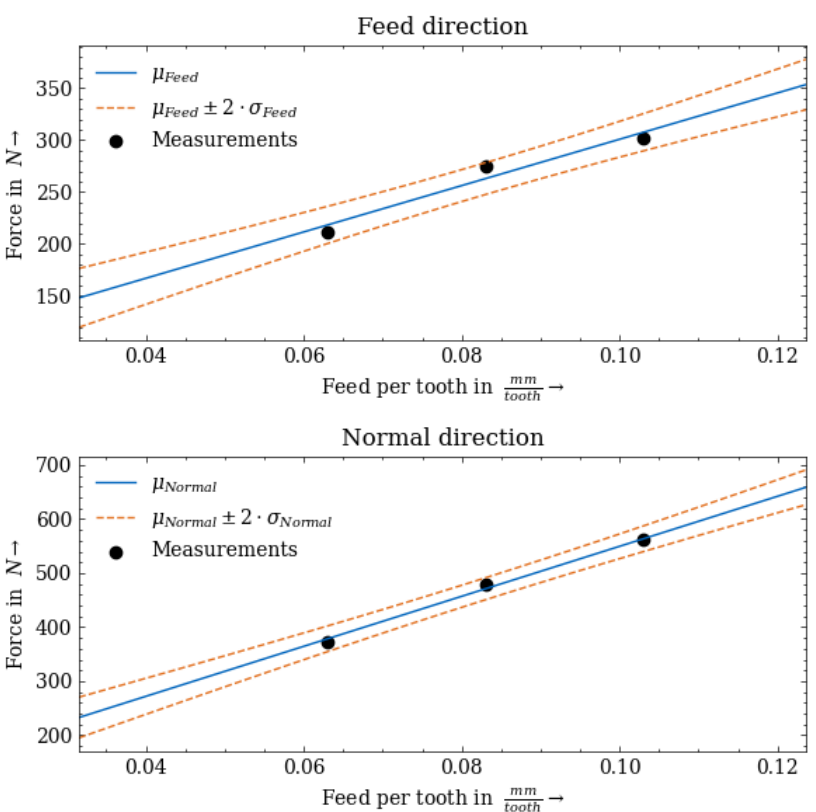

Figure 4: Bayesian linear regression using conjugate priors and three cutting force measurements.

The mean and the $95 \%$ credible intervals of the estimated, conditional cutting force parameters are provided in Table 1. The covariance matrices of the conditional joint probability distributions of $\boldsymbol{\beta}_{\text {Feed }}$ and $\boldsymbol{\beta}_{\text {Normal }}$ are as follows:

$\boldsymbol{V}_{\boldsymbol{\beta}_{\text {Feed }}}^{*}=\left[\begin{array}{cc}11049.39 & -720.29 \\ -720.29 & 48.82\end{array}\right]$,
$\boldsymbol{V}_{\boldsymbol{\beta}_{\text {Normal }}}^{*}=\left[\begin{array}{cc}19487.97 & -1270.38 \\ -1270.38 & 86.10\end{array}\right]$.

Table 1: Conditional means and $95 \%$ credible intervals of the estimated cutting force coefficient distributions

\begin{tabular}{ccc}
\hline & Mean & 95\% credible interval (Cl) \\
\hline$k_{t}$ & $2057.29 \frac{\mathrm{N}}{\mathrm{mm}^{2}}$ & {$[1783.68,2330.90] \frac{\mathrm{N}}{\mathrm{mm}^{2}}$} \\
$k_{t e}$ & $30.28 \frac{\mathrm{N}}{\mathrm{mm}}$ & {$[12.10,48.47] \frac{\mathrm{N}}{\mathrm{mm}}$} \\
$k_{n}$ & $991.53 \frac{\mathrm{N}}{\mathrm{mm}^{2}}$ & {$[785.50,1197.55] \frac{\mathrm{N}}{\mathrm{mm}^{2}}$} \\
$k_{n e}$ & $27.08 \frac{\mathrm{N}}{\mathrm{mm}}$ & {$[13.39,40.78] \frac{\mathrm{N}}{\mathrm{mm}}$} \\
\hline
\end{tabular}

\section{UNCERTAINTY PROPAGATION TO THE STABILITY ANALYSIS}

The estimated probability distributions of the cutting force coefficients can be used to quantify the resulting uncertainty in the stability analysis.

Within this publication, the stability analysis is based on the Zero Order Approximation (ZOA) approach by Altintas and Budak [Altintas et al. 1995] (see section 2).

This forward uncertainty quantification process is usually conducted using simple Monte Carlo simulations, as described in section 2. Since Monte Carlo simulations represent a large number of random computer experiments (making use of the Law of Large Numbers [Sullivan 2015]) and as defined by the central limit theorem, a Monte Carlo simulation converges with $\frac{1}{\sqrt{n_{M C}}}$, with $n_{M C}$ being the number of random Monte Carlo samples. Consequently, a very large number of Monte Carlo runs is needed to provide a reliable estimation of the stability limits' mean and distribution properties.

There are different approaches to reduce the computation effort without reducing the precision of the uncertainty estimates, namely quasi Monte Carlo (QMC) approaches and polynomial chaos expansion (PCE) methods. In this work, both approaches (QMC and PCE) are used to estimate the resulting stability limit and its uncertainty based on the estimated probability distributions of the cutting force coefficients. Additionally, their computational efficiency is evaluated and compared.

Quasi Monte Carlo (QMC) approaches increase the sample coverage over the input domain. Thus, fewer samples are needed compared to regular Monte Carlo methods [Tennøe et al. 2018]. Following the methodology of QMC approaches, a predefined number of evenly random parameter samples of $k_{t}$ and $k_{n}$ are drawn and evaluated in the subsequent stability analysis. Nonetheless, there are still many evaluations of the stability model needed.

In contrast to regular or quasi Monte Carlo approaches, polynomial chaos expansion (PCE) methods approximate the underlying model by polynomial functions [Tennøe et al. 2018]. Following the methodology of Tennøe et al. for the uncertainty propagation, the uncertainty arising in the stability model $b_{\text {lim }}\left(\boldsymbol{\gamma}, k_{t}, k_{n}\right)$ based on deterministic inputs $\gamma$ and the uncertain cutting force coefficients $k_{t}$ and $k_{n}$, the model is approximated by a polynomial expansion $\hat{b}_{\text {lim }}$ [Tennøe et al. 2018]:

$$
b_{\text {lim }} \approx \hat{b}_{\text {lim }}\left(\boldsymbol{\gamma}, k_{t}, k_{n}\right)=\sum_{n=0}^{N_{p}-1} c_{n}(\boldsymbol{\gamma}) \cdot \phi\left(k_{t}, k_{n}\right) .
$$

The number of expansion factors $N_{p}$ can be calculated as

$$
N_{p}=\left(\begin{array}{c}
d+p \\
p
\end{array}\right) \text {, }
$$

where $d$ is the number of uncertain parameters (in this case $d=2$ ) and $p$ equals the polynomial order [Tennøe et al. 2018]. The type of the polynomials $\phi\left(k_{t}, k_{n}\right)$ is chosen based on the type of the uncertain parameters, whereas the expansion coefficients $c_{n}(\gamma)$ are estimated using numerical regression algorithms. Detailed descriptions on the theory and numerical implementations of PCE methods are presented in [Feinberg et al. 2015; Tennøe et al. 2018; Yang et al. 2017].

The calculated stability limit was validated using multiple cutting experiments, each with half immersion but with different process parameters, namely with different spindle speeds and different axial depths of cut. The process stability was evaluated using the autocorrelation coefficient $R_{y y}$ as proposed by Zaeh et al. [Zaeh et al. 2020]. The autocorrelation coefficient $R_{y y}$ evaluates the self-similarity of the workpiece acceleration $a_{y}(t)$ close to the machining process. Based on empirical studies, it was determined, that an autocorrelation coefficient $R_{y y}<0.7$ characterizes an unstable process, even though this coefficient can also be used as a continuous chatter metric.

The test setup is illustrated in Figure 5. 


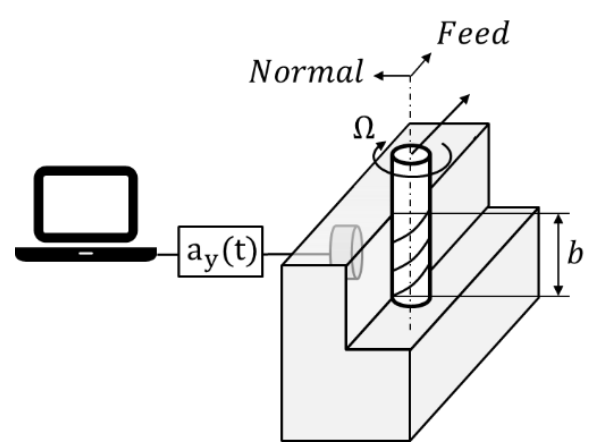

Figure 5: Experimental setup.

The stability analysis is not only dependent on the cutting force coefficients $k_{t}$ and $k_{n}$, but also on the relative workpiece-cutter dynamics. The frequency response function (FRF) in normal direction was averaged using five impact hammer tests, whereas the FRF in feed direction was neglected due to a significantly lower compliance in feed direction. The averaged FRF in normal direction is shown in Figure 6.

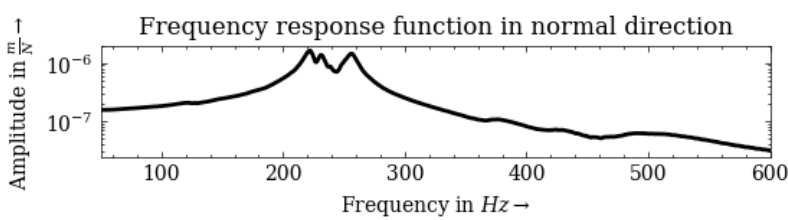

Figure 6: Averaged frequency response function between $50 \mathrm{~Hz}$ and $600 \mathrm{~Hz}$, as the relevant dynamics are present within this range.

As the FRF was averaged from five measurements, it is assumed to be deterministic without any uncertainty. Hence, the FRF was included in the deterministic model inputs $\gamma$. It is worth mentioning, that this assumption neglects uncertainties which might occur due to temperature-dependant phenomena or nonlinear friction effects. Possible future developments to overcome this issue are presented in section 6 .

The computed stability lobes using PCE $\left(\mu_{P C E}\right)$ and QMC $\left(\mu_{Q M C}\right)$ are shown in Figure 7. Additionally, the prediction uncertainty is illustrated in form of the estimated $95 \%$ credible interval $(\mathrm{Cl})$ for both approaches $\left(95 \% C I_{P C E}\right.$ and $\left.95 \% C I_{Q M C}\right)$.

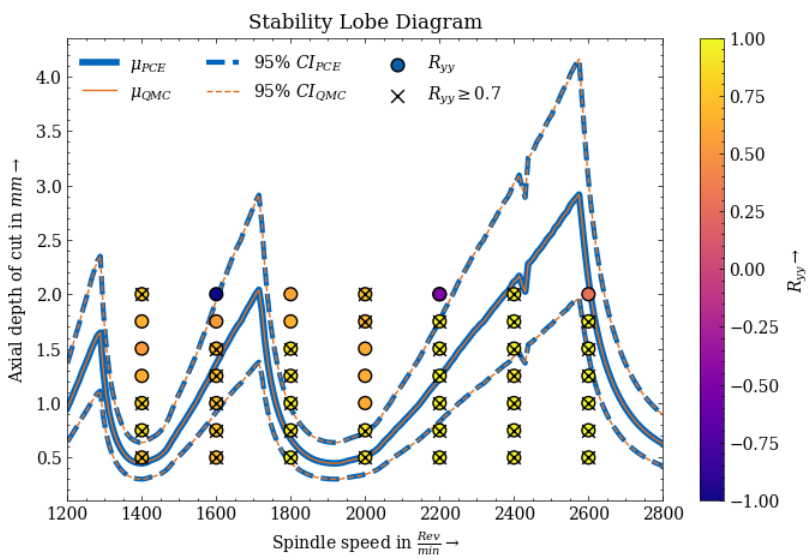

Figure 7: Stability lobe diagram including the uncertainty estimates using PCE and QMC.
It is clearly visible, that the uncertainty estimation based on PCE yields the same results as the computation using QMC.

Furthermore, the resulting autocorrelation coefficient for 49 cutting experiments is presented in Figure 7 . The results indicate, that the overall process stability is captured using the estimated mean (and thus the conventional deterministic approach). However, the estimated uncertainty enables a more robust and more reliable process design, as the lower credible bound always ensures a stable process.

\section{IMPLEMENTATION DETAILS}

All computations were performed on a conventional computer (Intel Core ${ }^{\mathrm{TM}}$ i7 CPU with $1.80 \mathrm{GHz}$ ). The program code was written in the programming language Python 3.

As the posteriors of the Bayesian cutting force parameter identification were computed analytically instead of using MCMC methods, the computation was performed in less than $15 \mathrm{~ms}$

The implementation of the uncertainty propagation method is primarily based on the uncertainty quantification frameworks Chaospy [Feinberg et al. 2015] and Uncertainpy [Tennøe et al. 2018].

A particular advantage of the PCE-based uncertainty propagation is the significantly faster computation time as visualized in Figure 8 . The PCE computation is by a factor of almost 265 faster than the QMC approach.

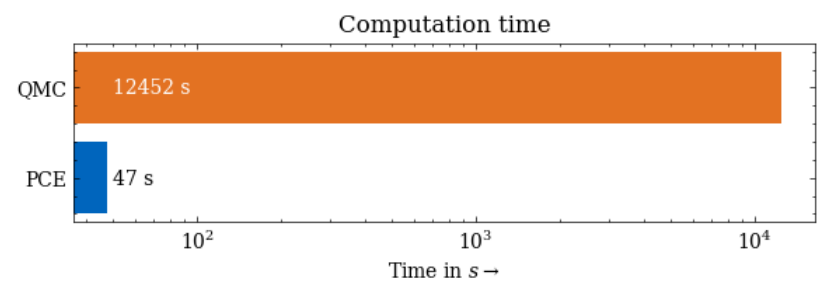

Figure 8: Comparison of computation times for the uncertainty propagation.

The configuration parameters of both evaluated approaches are listed in Table 2.

Table 2: Computation settings of PCE and QMC

\begin{tabular}{ccc}
\hline & Parameter & Value \\
\hline $\boldsymbol{U}$ & Method & CoLLOCATION \\
\hline & Polynomial order & 4 \\
\hline \multirow{O}{U}{} & Number of samples & $10^{4}$ \\
\hline
\end{tabular}

\section{DISCUSSION}

It is apparent, that the presented methodology, consisting of an analytical estimation of the cutting force coefficient's probability distributions and a PCE-based uncertainty propagation for the stability analysis, is able to reduce the computation times significantly in comparison to published approaches. Additionally, the presented methodology indicates, that the resulting probability distributions of the 
stability limits are not normally distributed as assumed in related works, but are rather log-normally distributed. Figure 9 illustrates the resulting probability distribution of the stability limit at $2300 \frac{\mathrm{rev}}{\mathrm{min}}$. Hence, it is worth noting, that as the probability distribution is clearly not symmetric, the estimated mean does not represent the maximum of the probability density.

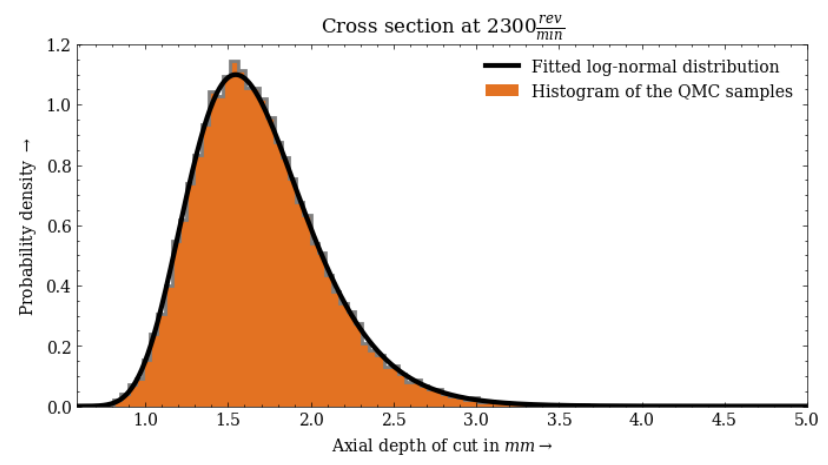

Figure 9: The probability distribution of the stability limit at $2300 \mathrm{rev} / \mathrm{min}$.

Furthermore, the current work only considers the cutting force coefficients as random variables. This assumption is valid, as the dynamics are considered by measured frequency response functions at the location of the machining process. Nonetheless, measured frequency responses functions may be prone to errors and uncertainties as well. Hence, the uncertainties arising from uncertain structural dynamics should be accounted for in the future. The authors intend to pursue this approach by applying Bayesian inference techniques to model the uncertainty in the structural dynamics model as as well, e.g. as presented in [Busch et al. 2020].

\section{SUMMARY}

In this publication, a two-step-methodology has been presented to quantify the uncertainty in the model-based stability analysis of milling processes: the uncertainty, which arises during the cutting force coefficient identification due to a limited number of cutting force experiments, was quantified using the analytic solution of a conjugate Bayesian regression model. Secondly, the coefficients' uncertainty was propagated to the stability analysis using a polynomial chaos expansion approach, which proves to be significantly faster than conventional (quasi) Monte Carlo simulations.

\section{REFERENCES}

[Altintas 2001] Altintas, Y. 2001. Analytical Prediction of Three Dimensional Chatter Stability in Milling. JSME International Journal Series C. 44(3):717-23

[Altintas et al. 1995] Altintas, Y.; Budak, E. 1995. Analytical Prediction of Stability Lobes in Milling. CIRP Annals Manufacturing Technology. 44(1):357-62

[Bhattacharyya et al. 2021] Bhattacharyya, A.; Schueller, J.; Mann, B.; Schmitz, T.; Gomez, M. 2021. Uncertainty propagation through an empirical model of cutting forces in end milling. Journal of Manufacturing Science and Engineering. 143(July):1-14
[Busch et al. 2020] Busch, M.; Schnoes, F.; Semm, T.; Zaeh, M.F.; Obst, B.; Hartmann, D. 2020. Probabilistic information fusion to model the pose-dependent dynamics of milling robots. Production Engineering. 14(4):435-44

[Duncan et al. 2005] Duncan, G.S.; Kurdi, M.; Schmitz, T.L.; Snyder, J. 2005. Stability lobe uncertainty. Proceedings of the 20th Annual ASPE Meeting, ASPE 2005. (January 2015):4-8

[Feinberg et al. 2015] Feinberg, J.; Langtangen, H.P. 2015. Chaospy: An open source tool for designing methods of uncertainty quantification. Journal of Computational Science. 11:46-57

[Karandikar et al. 2020] Karandikar, J.; Honeycutt, A.; Smith, S.; Schmitz, T. 2020. Milling stability identification using Bayesian machine learning. Procedia CIRP. 93:1423-28

[Karandikar et al. 2014] Karandikar, J.M.; Schmitz, T.L.; Abbas, A.E. 2014. Application of Bayesian Inference to Milling Force Modeling. Journal of Manufacturing Science and Engineering. 136(2):021017

[Li et al. 2020] Li, K.; He, S.; Liu, H.; Mao, X.; Li, B.; Luo, B. 2020. Bayesian uncertainty quantification and propagation for prediction of milling stability lobe. Mechanical Systems and Signal Processing. 138:106532

[Salehi et al. 2019] Salehi, M.; Schmitz, T.L.; Copenhaver, R.; Haas, R.; Ovtcharova, J. 2019. Probabilistic sequential prediction of cutting force using kienzle model in orthogonal turning process. Journal of Manufacturing Science and Engineering, Transactions of the ASME. 141(1):1-12

[Schmitz et al. 2019] Schmitz, T.L.; Smith, K.S. 2019. Machining Dynamics. Cham: Springer International Publishing

[Schmucker et al. 2021] Schmucker, B.; Trautwein, F.; Semm, T.; Lechler, A.; Zaeh, M.F.; Verl, A. 2021. Implementation of an Intelligent System Architecture for Process Monitoring of Machine Tools. Procedia CIRP. 96:342-46

[Sullivan 2015] Sullivan, T.J. 2015. Introduction to Uncertainty Quantification, Vol. 63. Cham: Springer International Publishing

[Tennøe et al. 2018] Tennøe, S.; Halnes, G.; Einevoll, G.T. 2018. Uncertainpy: A Python Toolbox for Uncertainty Quantification and Sensitivity Analysis in Computational Neuroscience. Frontiers in Neuroinformatics. 12(August):1-29

[Yang et al. 2017] Yang, S.; Xiong, F.; Wang, F. 2017. Polynomial Chaos Expansion for Probabilistic Uncertainty Propagation. In Uncertainty Quantification and Model Calibration, Vol. 32, 137-44. InTech

[Zaeh et al. 2020] Zaeh, M.F.; Schnoes, F.; Obst, B.; Hartmann, D. 2020. Combined offline simulation and online adaptation approach for the accuracy improvement of milling robots. CIRP Annals. 69(1):337-40 